\title{
State Resolved Thermochemical Modeling of Nitrogen Using DSMC
}

\author{
Jae Gang Kim* and Iain D. Boyd ${ }^{\dagger}$ \\ University of Michigan, Ann Arbor, MI 48109, USA
}

\begin{abstract}
State-resolved analyses of $\mathrm{N}+\mathrm{N}_{2}$ are performed using the direct simulation Monte Carlo (DSMC) method. In describing the elastic collisions in a state-resolved method, a statespecific total cross section is proposed. The state-resolved model is constructed from the state-specific total cross section and the state-to-state transition cross sections for boundbound and bound-free transitions taken from a NASA database. This approach makes it possible to analyze the rotation-to-translation, vibration-to-translation, and rotation-tovibration energy transfer and the chemical reactions without relying on a phenomenological model. In nonequilibrium heat bath and 2-D cylindrical flow, the DSMC calculations by the state-resolved model are compared with those obtained with previous DSMC models and master equation calculations. In these previous DSMC models, the VSS, phenomenological LB, QK, and TCE models are considered. From these studies, it is concluded that the present state-resolved model more accurately describes the rotational and vibrational relaxation and chemical processes than the other previous DSMC models.
\end{abstract}

\section{Nomenclature}

$\sigma_{b}, \sigma_{f}$ State-to-state transition cross sections for bound-bound and -free transitions, respectively, $\mathrm{cm}^{2}$

$\sigma_{T} \quad$ Total cross section, $\mathrm{cm}^{2}$

$D \quad$ Dissociation energy of $\mathrm{N}_{2}, \mathrm{erg}$

e $\quad$ Energy per particle, erg

$E_{c} \quad$ Total collision energy, erg

$E_{t r} \quad$ Relative translational energy, erg

$K \quad$ State-to-state transition rate coefficient, $\mathrm{cm}^{3} \mathrm{sec}^{-1}$

$k \quad$ Boltzmann constant, $\mathrm{erg} / \mathrm{K}$

$K_{f} \quad$ Dissociation rate coefficient, $\mathrm{cm}^{3} \mathrm{sec}^{-1}$

$K_{p} \quad$ Predissociation rate coefficient, $\sec ^{-1}$

$m_{r} \quad$ Reduced mass, $g$

$n \quad$ Number density, $\mathrm{cm}^{-3}$

$t \quad$ Time, sec

$T, T_{r}, T_{v}$ Translational, rotational, and vibrational temperatures, respectively, $K$

$v, J \quad$ Vibrational and Rotational states of a molecule, respectively

Subscripts

$\mathrm{N}, \mathrm{N}_{2} \quad$ Atomic and molecular nitrogen

\section{Introduction}

$T^{\mathrm{N}}$ Earth re-entry conditions from the Moon and Mars, the speed of a returning capsule is about $10 \mathrm{~km} / \mathrm{sec}$ 1 to $16 \mathrm{~km} / \mathrm{sec}$ in the rarefied gas regime. In these high-speed return cases, complicated thermochemical

*Post-doctoral Research Fellow, Department of Aerospace Engineering, 1320 Beal Avenue, Member of AIAA.

$\dagger$ Professor, Department of Aerospace Engineering, 1320 Beal Avenue, AIAA Fellow. 
nonequilibrium phenomena of strong ionization, high radiative heating, and high ablation rates of thermal protection system may occur. One of the complicated thermochemical processes in these high-speed return cases is rotational nonequilibrium of $\mathrm{N}_{2}$. In the shock-tube experiments by Sharma and Gillespie ${ }^{1}$ and Fujita et al. ${ }^{2}$ for $\mathrm{N}_{2}$ and air species, the behavior of rotational relaxation in the high-speed re-entry conditions and high-temperature environment were studied spectroscopically. In the pioneering work by Parker $^{3}$ and Lordi and Mates, ${ }^{4}$ the effective collision number of the rotational mode of $\mathrm{N}_{2}$ is of the order of 10 . This means that the rotational model is expected to equilibrate with the translation mode within about 10 collisions. However, in these shock-tube experiments, ${ }^{1,2}$ the measured rotational temperature was close to the vibrational temperature. This result implies that the effective collision number of rotational relaxation is much larger than 10. In this larger effective collision number of rotational relaxations, the rotation-tovibration energy transfer is important. In reality, rotational excitation of a molecule by collisions with heavy particles occurs partly by the transfer of translational energy and partly by the transfer of vibrational energy. At small effective collision numbers, the rotational excitation by the transfer of the translational energy is dominant and the other transfer of vibrational energy is ignored. However, at large effective collision numbers, the rotational excitation by the transfer of vibrational energy should be considered. In DSMC, there exist limitations to describe the rotation-to-vibration energy transfer by heavy-particle collisions. The energy transitions in DSMC are of a phenomenological nature, and these models describe the inelastic collisions and chemical reactions at the macroscopic level. In addition, the rotation-to-vibration energy transfer is difficult to describe in phenomenological models, such as the Larsen-Borgnakke (LB) model. ${ }^{5}$ Only the energy transitions of rotation-to-translation and vibration-to-translation are available.

For $\mathrm{N}+\mathrm{N}_{2}$, the complete sets of state-to-state transition cross sections were recently calculated by Jaffe et al. ${ }^{6,7}$ The quasi-classical trajectory method was adopted to calculate these transition cross sections based on new $\mathrm{N}_{3}$ potential energy surfaces. In the present work, the thermochemical nonequilibrium of $\mathrm{N}+\mathrm{N}_{2}$ by a state-resolved method in the DSMC is studied by using these state-to-state transition cross sections. In describing the elastic and inelastic collisions at the molecular level, a state-specific total cross section is proposed. The rotation-to-translation, vibration-to-translation, and rotation-to-vibration energy transfer and nonequilibrium chemical reactions are considered by adopting the state-specific total cross section model and state-to-state transition cross sections, that do not rely on a phenomenological approach, in 0-D heat bath and 2-D cylindrical flow calculations. The results of the present state-resolved DSMC calculations are compared with the results of master equation calculations and previous phenomenological DSMC methods.

\section{State-specific total cross sections for atom-molecule collision}

Previously, the variable hard sphere (VHS), ${ }^{5}$ variable soft sphere (VSS) ${ }^{8,9}$ general hard sphere (GHS), ${ }^{10}$ and general soft sphere (GSS ${ }^{11,12}$ models were developed to determine a total cross section in DSMC. In these previous models, the collision parameters are determined at the macroscopic level by the curve-fit of the transport properties and collision integrals. The previous models have an assumption that the total cross section depends only on the relative translational energy between colliding particles. This assumption is valid in low temperature gases where the high-rotational and vibrational states of molecules are not excited. When the high rotational and vibrational states of molecules are excited, however, this assumption is not valid. For these high energy states, the inter-nuclear distance of the colliding molecules is large and significantly influences the total cross section.

In the present work, a state-specific total cross section (SST) for each rotational and vibrational state is proposed to address these uncertainties. This SST model for atom-molecule collision is defined as follows:

$$
\sigma d \Omega=b d b d \epsilon
$$

where $b$ is the impact parameter, $\epsilon$ is the angle between the reference and collision planes. The differential solid angle of post-collision $d \Omega$ is defined as

$$
d \Omega=\sin \chi d \chi d \epsilon
$$

where $\chi$ is the scattering angle. Then, the total cross section can be derived from Eqs. (1) and (2).

$$
\sigma_{T}\left(E_{t r}\right)=\int \sigma d \Omega=2 \pi \int \sigma \sin \chi d \chi=2 \pi \int b d b .
$$


Table 1. Collision parameters of the VHS, VSS, and GSS models for $\mathbf{N}+\mathbf{N}_{2}$.

\begin{tabular}{ccc|cc} 
& $\mathrm{VHS}^{15,16}$ & $\mathrm{VSS}^{9}$ & & $\mathrm{GSS}^{12}$ \\
\hline$\sigma_{T_{\text {ref }}}\left(A^{2}\right)$ & 35.15 & 32.85 & $C_{1}\left(A^{2} / J\right)$ & -23.02 \\
$T_{\text {ref }}(K)$ & 1,000 & 1,000 & $C_{2}\left(A^{2} / J\right)$ & $8.721 \times 10^{-4}$ \\
$\omega$ & 0.665 & 0.775 & $\omega_{1}$ & $-2.928 \times 10^{-2}$ \\
$C_{\alpha}$ & 1.0 & 1.495 & $\omega_{2}$ & 0.241 \\
$\omega_{\alpha}$ & 0.0 & 0.0435 & $\alpha$ & 1.7826
\end{tabular}

In the definition of the cross section, the total cross section can be expressed classically as the product of a geometrical cross section $\pi b_{\max }^{2}$ and an averaged probability of collision $P\left(E_{t r}\right)$, i. e.,

$$
\begin{gathered}
\sigma_{T}\left(E_{t r}\right)=2 \pi \int b d b=\pi b_{\max }^{2} P\left(E_{t r}\right), \\
P\left(E_{t r}\right)=\frac{1}{(2 \pi)^{3} b_{\max }^{2}} \int_{b=0}^{b_{\max }} \int_{\theta=0}^{\pi} \int_{\phi=0}^{2 \pi} p\left(E_{t r}, b, \theta, \phi\right) b \sin \theta d b d \theta d \phi,
\end{gathered}
$$

where $p\left(E_{t r}, b, \theta, \phi\right)$ is a trajectory-computed probability that the collision occurs. These Eqs. (4) and (5) only treat the atom-atom collision. Now, we extend these Eqs. (4) and (5) to treat the atom-molecule collision. These extended equations have the form

$$
\begin{gathered}
\sigma_{T}\left(E_{t r}\right)=2 \pi \int b d b=\pi b_{\text {max }}^{2} P\left(E_{t r}, v, J\right), \\
P\left(E_{t r}, v, J\right)=\frac{1}{(2 \pi)^{3} b_{\text {max }}^{2}} \int_{b=0}^{b_{\max }} \int_{\theta=0}^{\pi} \int_{\phi=0}^{2 \pi} \int_{R=\rho^{-}}^{\rho^{+}} \int_{\eta=0}^{2 \pi} p\left(E_{t r}, b, \theta, \phi, R, \eta\right) \operatorname{bsin} \theta d b d \theta d \phi d R(v, J) d \eta(J),
\end{gathered}
$$

where the inter-nuclear distance $R$ is a dependent variable of the rotational and vibrational states $(v, J)$. The initial orientation of the angular momentum of the molecule $\eta$ is dependent on rotational state $J$. As seen in Eqs. (6) and (7), the SST model depends on the rotational and vibrational states of molecules and it is determined at the microscopic level based on the real potential energy surface.

For $\mathrm{N}+\mathrm{N}_{2}$, the total cross section by the SST model is calculated in the present work by a quasi-classical trajectory (QCT) method using the recently calculated potential energy surface of $\mathrm{N}_{3}$ by Jaffe et al. ${ }^{6,7} \mathrm{~A}$ stratified sampling is used in obtaining the impact parameter. All other parameters are Monte Carlo-selected in the QCT calculations. A total of 2,000 trajectories are calculated per impact parameter with a batch size of $0.1 \mathrm{~A}$. Relative translational energies of $0.5 \mathrm{eV}$ to $50 \mathrm{eV}$ are considered in the trajectory calculations. Further details about the QCT calculations can be found in other literature. ${ }^{13,14}$

In Fig. 1, the total cross section of the SST model is compared with the VHS, VSS, and GSS models. In the VHS and VSS models, the total cross section is calculated as

$$
\sigma_{T}=\sigma_{T_{r e f}} \frac{\left(k T_{r e f} / E_{t r}\right)^{\omega-0.5}}{\Gamma(2.5-\omega)}
$$

and the scattering parameter $\alpha$ is defined as

$$
\alpha=C_{\alpha}\left(E_{t r} / k\right)^{\omega_{\alpha}},
$$

where the collision parameters $\sigma_{r e f}, T_{r e f}, \omega, C_{\alpha}$, and $\omega_{\alpha}$ of the VHS model are obtained from the curve fit results of high-temperature viscosity data for $\mathrm{N}+\mathrm{N}$ by Svehla ${ }^{15}$ and $\mathrm{N}_{2}+\mathrm{N}_{2}$ by Stallcop et al., ${ }^{16}$ and the collision parameters of the VSS models are obtained from the curve fit results of diffusion and viscosity collision integrals for $\mathrm{N}+\mathrm{N}_{2}$ by Koura and Matsumoto. ${ }^{9}$ In the present work, these collision parameters are reproduced to have the form of Eqs. (8) and (9). In the GSS model, the total cross sections are calculated by

$$
\sigma_{T}=C_{1} E_{t r}^{-\omega_{1}}+C_{2} E_{t r}^{-\omega_{2}}
$$


where the collision parameters $C_{1}, C_{2}, \omega_{1}$, and $\omega_{2}$ and scattering parameter $\alpha$ are obtained from the curve fit results of diffusion and viscosity collision integrals for $\mathrm{N}+\mathrm{N}_{2}$ by Kim et al. ${ }^{12}$ These collision parameters of the VHS, VSS, and GSS models are tabulated in Table 1. In the comparisons of the SST model for various rotational states of figures (a) and (b), the differences of the total cross sections of each rotational state are negligible. However, in the vibrational states of figures (c) and (d), these differences are discernible. These results show that the inter nuclear-distance of molecules in each rotational and vibrational state affects the total cross section in heavy-particle collisions and this total cross section is mostly dependent on the vibrational state of the molecule. In comparisons between the SST and previous models, discernible differences of the total cross section are shown in all figures. The total cross sections of the VSS and GSS models are 3.5 times smaller than the minimum cross section value of the SST model for $\mathrm{N}+\mathrm{N}_{2}$.

In $\mathrm{N}_{2}, 9,390$ rotational and vibrational states exit in the electronic ground state. The maximum vibrational state is 60 and the maximum rotational state is variable for each vibrational state from 2 to 279 . In describing the total cross section of $\mathrm{N}+\mathrm{N}_{2}$ by the SST model, we need 9,390 sets of total cross sections for each relative translational energy. However, the total cross section is mostly dependent on the vibrational state. Then, the sets of total cross sections can be reduced to 61 . The complete sets of the SST model for each vibrational state are taulated in the Appendix in the form of a curve-fit function:

$$
\sigma_{T}\left(E_{t r}, v\right)=\exp \left[a_{1}+a_{2} E_{t r}+a_{3} \ln \left(\frac{1}{E_{t r}}\right)+\frac{a_{4}}{E_{t r}}+\frac{a_{5}}{E_{t r}^{2}}\right],
$$

where $a_{1-5}$ are the curve-fit coefficients. In these tabulated data, $E_{t r}$ unit is $\mathrm{eV}$.

\section{State-resolved bound-bound transitions}

In the present work, a non-phenomenological approach is made for $\mathrm{N}+\mathrm{N}_{2}$ in describing the stateresolved energy transitions. The rotation-to-translation (R-T), vibration-to-translation (V-T), and rotationto-vibration (R-V) energy transfers are described by using the SST model and the complete sets of state-tostate transition cross sections calculated by Jaffe et al. ${ }^{6,7}$ In these sets of transition cross sections for $\mathrm{N}+\mathrm{N}_{2}$, the database was constructed for 9,390 rotational and vibrational states of the electronic ground state of $\mathrm{N}_{2}$. In these 9,390 rotational and vibrational states, truly bound and quasi-bound states were included. The QCT method was adopted to evaluate these cross sections for bound-bound, bound-free and exchange reactions. The database for the $\mathrm{N}+\mathrm{N}_{2}$ system comprises more than 23 million reactions and these transition cross sections were evaluated for the relative translational energy from $0.28 \mathrm{eV}$ to $18 \mathrm{eV}$. In describing the R-T, V-T, and R-V transitions without using a phenomenological approach, the state-to-state transition probabilities of atom-molecule collisions are determined as

$$
P_{b}\left(E_{t r} ; v, J \rightarrow v^{\prime} J^{\prime}\right)=\int_{v_{0}}^{v^{\prime}} d v^{\prime \prime} \int_{J_{0}}^{J^{\prime}} d J^{\prime \prime} \frac{\sigma_{b}\left(E_{t r} ; v, J \rightarrow v^{\prime \prime}, J^{\prime \prime}\right)}{\sigma_{T}\left(E_{t r}, v, J\right)} .
$$

In the present work, micro-reversibility is adopted in the state-to-state cross sections as

$$
\sigma\left(E_{t r} ; v, J \rightarrow v^{\prime}, J^{\prime}\right)(2 J+1) g_{s} m_{r} E_{t r}=\sigma\left(E_{t r} ; v^{\prime}, J^{\prime} \rightarrow v, J\right)\left(2 J^{\prime}+1\right) g_{s}^{\prime} m_{r} E_{t r}^{\prime} .
$$

In $\mathrm{N}_{2}$, the nuclear spin degeneracy $g_{s}$ is 6 when $J$ has an even number and 3 when an odd number. Then, the state-resolved model (SST+RVT) is constructed by the SST model and the state-to-state transition probabilities of Eq. (12)

The rotational and vibrational energy relaxations using the state-resolved model are studied in isothermal heat bath conditions. A zero-dimensional DSMC code ${ }^{5}$ with some manipulations is used in the present work. In the DSMC calculations, 300,000 particles are generated. Initial number densities of $\mathrm{N}$ and $\mathrm{N}_{2}$ are set to a constant of $5 \times 10^{17} \mathrm{~cm}^{-3}$, and initial conditions of equilibrium and nonequilibrium temperatures are tabulated in Table 2. In the present work, the initial rotational and vibrational energy of $\mathrm{N}_{2}$ at temperature $T$ is generated by using the probability of partition function relations as

$$
P_{v, J}=\frac{Q_{v, J}}{\sum_{v, J} Q_{v, J}}
$$

where $Q$ is the rovibrational partition function defined as

$$
Q_{v, J}=\psi g_{e}(2 J+1) g_{s} \exp \left(-\frac{e_{v, J}}{k T}\right),
$$


Table 2. Initial heat bath conditions for study of bound-bound transitions.

\begin{tabular}{ccc} 
Case & $T(K)$ & $T_{r}=T_{v}(K)$ \\
\hline C1 & 6,000 & 2,000 \\
C2 & 8,000 & 2,000 \\
C3 & 10,000 & 2,000 \\
C4 & 20,000 & 2,000 \\
C5 & 30,000 & 2,000 \\
C6 & 40,000 & 2,000 \\
C7 & 50,000 & 2,000 \\
C8 & 70,000 & 2,000
\end{tabular}

where $\psi$ is a symmetric factor of the molecule and $g_{e}$ is the degeneracy of the electronic state.

In validating the results of the DSMC calculations in heat bath conditions, master equation calculations are used. The master equation of bound-bound transitions is defined as follows;

$$
\frac{d n_{v, J}}{d t}=\sum_{v, J} K\left(v, J \rightarrow v^{\prime}, J^{\prime}\right) n_{x}\left[\frac{Q_{v, J}}{Q_{v^{\prime}, J^{\prime}}} n_{v^{\prime}, J^{\prime}}-n_{v, J}\right]
$$

where $n_{x}$ is the number density of the colliding species. The principle of detailed balance between the forward and backward rates is invoked under equilibrium, which leads to

$$
K\left(v, J \rightarrow v^{\prime}, J^{\prime}\right) Q_{v, J}=K\left(v^{\prime}, J^{\prime} \rightarrow v, J\right) Q_{v^{\prime}, J^{\prime}}
$$

The state-to-state transition rates $K$ are obtaind from the NASA database of Jaffe et al., ${ }^{6,7}$ which is the same reference database of the state-to-state transition cross sections used in the present work. The master equation of Eq. (16) is solved for initial conditions of the C3 to C5 cases.

In Fig. 2, relaxations of rotational and vibrational temperature and normalized averaged energies of the DSMC calculations by the SST+RVT approach are compared with those of the master equation calculations. In the present work, energy-equivalent rotational and vibrational temperatures are adopted to characterize the rotational and vibrational modes. The average energies of the DSMC and master equation calculations are normalized by the equilibrium energy specified by equilibrium temperature $T$. In figures (a) and (b), the temperature and averaged energy relaxation curves of the DSMC calculations by SST + RVT are almost identical to those of the master equation calculations.

In Fig. 3, normalized rotational and vibrational number density distributions in each relaxation process of C5 case are presented. The normalizations are performed by using the equilibrium number densities specified by equilibrium temperature $T$. In all procedures of convergence to equilibrium state, the distributions of the rotational and vibrational number densities of the SST + RVT agree well with the distributions of the master equation calculations. These results show that the R-T, V-T, and R-V transitions of $\mathrm{N}+\mathrm{N}_{2}$ are accurately simulated by the state-resolved model of SST + RVT in DSMC calculations.

In Fig. 4, the characteristic relaxation parameters of $p \tau_{r}$ and $p \tau_{v}$ calculated in the present DSMC model are compared with Park's model ${ }^{17}$ for $\mathrm{N}+\mathrm{N}_{2}$. In the present work, the relaxation times of the rotational and vibrational modes are determined by using the Landau-Teller form ${ }^{18}$ with the e-folding collision number method. By multiplying by the pressure, one obtains the characteristic rotational and vibrational relaxation parameters $p \tau_{r}$ and $p \tau_{v}$, respectively. The figure shows that the rotational relaxation time is faster than that of vibration initially. However, the differences of relaxation time between the rotational and vibrational modes become small when the temperature increases. At temperatures above $30,000 \mathrm{~K}$, the rotational and vibrational relaxation times are almost identical. These results show that, in $\mathrm{N}+\mathrm{N}_{2}$, modeling of $\mathrm{R}-\mathrm{V}$ transitions in DSMC is important when the temperature increases. In comparison of vibrational relaxation with Park's model and the collision limiting corrections ${ }^{17}$ by $\sigma_{v}=3.0 \times 10^{-17} \mathrm{~cm}^{2}$, discernable differences are observed, especially at temperatures above 2,000K. Park's model is based on the empirical equation proposed by Millikan and White, ${ }^{19}$ while the present DSMC results are evaluated by a state-resolved model, that is based on the recent $\mathrm{N}_{3}$ potential energy surfaces ${ }^{6}$ calculated by an $a b$-initio method. 
In the present work, comparisons are made between the results of DSMC calculations using the present state-resolved model and previous DSMC models for the $\mathrm{C} 3$ and $\mathrm{C} 4$ cases. In the previous models, the VSS model ${ }^{8,9}$ and the phenomenological LB model ${ }^{5}$ are adopted for total cross section and rotational and vibrational energy transfer, respectively. In the energy transfer of the LB models, the $\mathrm{R}-\mathrm{V}$ transition is ignored. This is because the $\mathrm{R}-\mathrm{V}$ transition is difficult to describe in the phenomenological method. The effective collision numbers for the R-T and V-T relaxations in the LB model are calculated by

$$
Z=p \tau \frac{\sigma_{T}}{\pi k T}\left(\frac{8 \pi k T}{m_{r}}\right)^{1 / 2},
$$

where the rotational and vibrational parameters $p \tau$ are obtained from the present results of Fig. 4 . The real 9,390 rotational and vibrational states of $\mathrm{N}_{2}$ are adopted in the LB model. Then, the comparisons of the DSMC calculations are performed for SST+RVT, VSS+RVT, and VSS+LB.

In Fig. 5, relaxations of rotational and vibrational temperatures and normalized average energies are compared between the DSMC calculations by SST+RVT, VSS+RVT, and VSS+LB. In VSS+RVT, the transition probability is calculated by Eq. (12) with substituting the total cross section of the SST model with the VSS model. In figures (a) and (b), it is shown that the rotational and vibrational temperatures and average energies of VSS+RVT under-predict the results of SST+RVT. The converged values of temperatures and average energies of the VSS+RVT do not approach the exact equilibrium values, even though microreversibility is adopted. In VSS+LB, the rotational and vibrational temperatures and average energies are slightly higher than the results of SST+RVT, and the results do not approach the equilibrium values.

In Fig. 6, comparisons of normalized rotational and vibrational number density distributions between the DSMC calculations by SST+RVT, VSS+RVT, and VSS+LB in each relaxation process of the C4 case are presented. In figures (a) and (b), it is observed that the rotational and vibrational number densities of VSS+RVT are less excited than those of SST+RVT. In VSS+LB, the number densities at high-rotational and vibrational energy levels above $J=100$ and $v=40$ are more excited than those by SST + RVT. In the comparisons with the Boltzmann distributions specified by nonequilibrium temperatures, strong nonequilibrium is observed in the rotational and vibrational number density distributions at $t=2.5 \times 10^{-9} \mathrm{sec}$, and this strong nonequilibrium is almost dissipated at $t=2.5 \times 10^{-8}$ sec. However, in VSS+LB, the number densities at the high-rotational and vibrational energies are still highly excited. These excited number density distributions of VSS+LB produce the over-predicted rotational and vibrational temperatures and average energies, as shown in Fig. 5.

Flows over a 2-D cylinder of $10 \mathrm{~cm}$ diameter are calculated by the SST+RVT, VSS+RVT, and VSS+LB models. The free-steam velocity is $10 \mathrm{~km} / \mathrm{sec}$ and the temperature is $200 \mathrm{~K}$. Number densities of $\mathrm{N}$ and $\mathrm{N}_{2}$ are set to a constant of $10 \times 10^{14} \mathrm{~cm}^{-3}$. The Knudsen number is about 0.2 based on the cylinder diameter and the total cross section of the SST model. In this 2-D cylindrical flow, the inelastic collisions of $\mathrm{N}+\mathrm{N}$ and $\mathrm{N}_{2}+\mathrm{N}_{2}$ are frozen to observe the rotational and vibrational nonequilibrium of $\mathrm{N}+\mathrm{N}_{2}$. In the elastic collision of $\mathrm{N}+\mathrm{N}_{2}$, the post-collision angle for SST+RVT is treated the same as the VHS model. ${ }^{5} 630,000$ particles are generated and the initial rotational and vibrational energies of molecules are determined by the partition function relations of Eq. (14).

In Fig. 7, comparisons of the flow velocity, species number densities, and temperatures along the stagnation line of the cylinder are presented. In the comparisons of species number densities of figure (a), there is no difference between SST+RVT, VSS+RVT, and VSS+LB. In the comparisons of flow velocity, the velocity of SST+RVT is higher and has a steeper gradient than that of VSS+RVT and VSS+LB near the cylinder surface. However, the difference of velocity between VSS+RVT and VSS+LB is small. The difference in velocity between SST and VSS arises from the moldeing of the total cross section. This difference of total cross section also affects the translational temperature, shown in figure (b). The translational temperature of SST+RVT is higher and changes rapidly near the cylinder surface than that of VSS+RVT and VSS+LB. However, the translational temperatures of VSS+RVT and VSS+LB are almost identical. In the comparisons of the rotational and vibrational temperatures, the rotational temperature is not higher than the vibrational temperature. These rotational and vibrational temperatures are almost identical in SST+RVT. In the comparisons between SST+RVT with VSS+RVT and VSS+LB, the rotational and vibrational temperatures of the VSS+RVT and VSS+LB are under-predicted compared to SST+RVT near the cylinder surface. 
Table 3. Initial heat bath conditions for study of bound-free transitions.

\begin{tabular}{ccc} 
Case & $T(K)$ & $T_{r}=T_{v}(K)$ \\
\hline C1 & 10,000 & 1,000 \\
C2 & 20,000 & 1,000 \\
C3 & 30,000 & 1,000 \\
C4 & 40,000 & 1,000 \\
C5 & 50,000 & 1,000 \\
C6 & 70,000 & 1,000
\end{tabular}

\section{State-resolved bound-free transitions}

In the present work, the chemical reactions of bound-free transitions are treated by a state-resolved method. The reaction probability of the state-resolved model is defined as

$$
P_{f}\left(E_{t r} ; v, J \rightarrow c\right)=\frac{\sigma_{f}\left(E_{t r} ; v, J \rightarrow c\right)}{\sigma_{T}\left(E_{t r}, v, J\right)},
$$

where the bound-free transition cross sections $\sigma_{f}$ of $\mathrm{N}+\mathrm{N}_{2}$ are obtained from the database of Jaffe et al. ${ }^{6,7}$ Then, the state-resolved model (SST $+\mathrm{RVT}+\mathrm{QCT}$ ) of $\mathrm{N}+\mathrm{N}_{2}$ is constructed by the SST model for total cross section and state-to-state cross sections to describe the R-T, V-T, and R-V energy transitions, and chemical reactions by using Eqs. (12) and (19), respectively. In this state-resolved model, the truly bound and quasibound states are considered in the bound-bound and bound-free transitions. However, predissociation of $\mathrm{N}_{2}$ is not considered, because its probability of occurrence is very small.

The rotational and vibrational energy relaxations and nonequilibrium chemical reactions of the stateresolved model of SST+RVT+QCT are studied in isothermal heat bath conditions. In DSMC calculations, 500,000 particles are generated. Initial number densities of $\mathrm{N}$ and $\mathrm{N}_{2}$ are set to a constant of $1 \times 10^{18} \mathrm{~cm}^{-3}$, and the initial equilibrium and nonequilibrium temperatures are tabulated in Table 3.

In validating the state-resolved DSMC calculations, master equation calculations are adopted. The master equation of bound-bound and bound-free transitions is defined as follows;

$$
\begin{aligned}
\frac{d n_{v, J}}{d t}= & \sum_{v^{\prime}, J^{\prime}} K\left(v, J \rightarrow v^{\prime}, J^{\prime}\right) n_{x}\left[\frac{Q_{v, J}}{Q_{v^{\prime}, J^{\prime}}} n_{v^{\prime}, J}-n_{v, J}\right] \\
& +K(v, J \rightarrow c) n_{x}\left[\frac{Q_{v, J} Q_{t_{N_{2}}}}{Q_{N}^{2} Q_{t_{N}}^{2}} \exp \left(\frac{D_{v, J}}{k T}\right) n_{N}^{2}-n_{v, J}\right] \\
& +K_{p}(v, J \rightarrow c)\left[\frac{Q_{v, J} Q_{t_{N_{2}}}}{Q_{N}^{2} Q_{t_{N}}^{2}} \exp \left(\frac{D_{v, J}}{k T}\right) n_{N}^{2}-n_{v, J}\right], \\
\frac{d n_{N}}{d t}= & 2 \sum_{v, J} K(v, J \rightarrow c) n_{x}\left[n_{v, J}-\frac{Q_{v, J} Q_{t_{N_{2}}}}{Q_{N}^{2} Q_{t_{N}}^{2}} \exp \left(\frac{D_{v, J}}{k T}\right) n_{N}^{2}\right] \\
& +2 \sum_{v, J} K_{p}(v, J \rightarrow c)\left[n_{v, J}-\frac{Q_{v, J} Q_{t_{N_{2}}}}{Q_{N}^{2} Q_{t_{N}}^{2}} \exp \left(\frac{D_{v, J}}{k T}\right) n_{N}^{2}\right],
\end{aligned}
$$

where the translational partition function $Q_{t}$ is defined as

$$
Q_{t}=V\left(\frac{m k T}{\hbar}\right)^{3 / 2},
$$

where $V$ is volume, $m$ is species mass, and $\hbar$ is Planck's constant. The principle of detailed balance relations for bound-free transitions are

$$
K(v, J \rightarrow c) \frac{Q_{v, J} Q_{t_{N_{2}}}}{Q_{N}^{2} Q_{t_{N}}^{2}} \exp \left(\frac{D_{v, J}}{k T}\right)=K(c \rightarrow v, J) .
$$


The state-to-state rates of bound-bound and bound-free transitions are obtained from the NASA database, which is the same reference database of the present state-to-state transition cross sections. The master equations of Eqs. (20) and (21) are calculated for the C1 to C3 cases of the heat bath conditions.

In Fig. 8, relaxations of rotational and vibrational temperatures, normalized average energies, and species number densities are presented for the DSMC calculations by SST+RVT+QCT and the master equation calculations. In figures (a) and (b), the rotational and vibrational temperatures and normalized energies of the DSMC calculations are almost the same as those of the master equation calculations before statistical fluctuations appear. These fluctuations of the temperatures and energies result from the dissociation of $\mathrm{N}_{2}$. In these periods, $\mathrm{N}_{2}$ is mostly dissociated and it is difficult to obtain statistically meaningful samples. In figures (a) and (b), the quasi-steady state (QSS) of rotational and vibrational energies is observed in both the DSMC and master equation calculations. In species number density relaxations of figure (c), the relaxations of $\mathrm{N}$ and $\mathrm{N}_{2}$ of the DSMC and master equation calculations are almost identical.

In Fig. 9, rotational and vibrational number density distributions in each relaxation process of the $\mathrm{C} 2$ case are presented for the DSMC calculation by SST+RVT+QCT and the master equation calculations. In figures (a) and (b), the number densities of the DSMC calculations have the same distributions as those of the master equation calculations. Especially, at $t=4 \times 10^{-8} \mathrm{sec}$ and $t=6 \times 10^{-8} \mathrm{sec}$, the QSS of rotational and vibrational states is clearly observed in the results of SST+RVT+QCT. The rotational and vibrational number density distributions are not changed in this time period, even though the distributions do not converge to the equilibrium states. These results show that the state-resolved model of SST+RVT+QCT can accurately describe the R-T, V-T, and R-V transitions and nonequilibrium chemical reactions.

In Fig. 10, the dissociation reaction rate coefficients obtained by SST+RVT+QCT are compared to the shock-tube measured data. ${ }^{20-23}$ In the present work, the reaction rate of dissociation in the QSS period is derived as follows;

$$
-\frac{d\left[N_{2}\right]}{d t}=K_{f}\left[N_{2}\right] n_{x}
$$

In comparisons with the shock-tube data, the calculated reaction rates by SST+RVT+QCT and master equation have similar values to the experiments by Appleton et al. ${ }^{22}$ A close fit to the calculated reaction rates by SST $+\mathrm{RVT}+\mathrm{QCT}$ is found in Arrhenius form as

$$
K_{f}=6.255 \times 10^{18} T^{-0.8351} \exp \left(\frac{113,200}{T}\right) \mathrm{cm}^{3} \mathrm{~mole}^{-1} \mathrm{sec}^{-1} .
$$

In the present work, the comparisons are made between the DSMC calculations by the state-resolved model and the previous DSMC models for the $\mathrm{C} 1$ and $\mathrm{C} 2$ cases of heat bath conditions. For the previous models, $\mathrm{TCE}^{5}$ and $\mathrm{QK}^{24}$ models are considered for the chemical reactions. In the QK models, the chemical reactions are tightly coupled with the phenomenological LB model, and the dissociation probability is determined by

$$
p_{D_{Q K}}=\left(1-i^{*} k \Theta_{v}\right)^{3 / 2-\omega},
$$

where $i^{*}$ is post-collision state which is chosen uniformly from the states equal to or below $i_{\max }=E_{c} /\left(k \Theta_{v}\right)$. In the present work, the characteristic vibrational temperature is set to a constant of $\Theta_{v}=1886.4 \mathrm{~K}$. This is the average characteristic vibrational temperature of $\mathrm{N}_{2}$, which is calculated by $\Theta_{v}=\left[e_{v}(60,0)-\right.$ $\left.e_{v}(0,0)\right] / 60 k$. In the TCE model, the chemical reactions are implicitly coupled with the VHS or VSS model and dissociation reaction rates of the Arrhenius form. The dissociation probability of the TCE model is defined as

$$
p_{D_{T C E}}=\frac{\pi^{1 / 2} \psi a T_{r e f}^{b}}{2 \sigma_{T_{\text {ref }}}\left(k T_{r e f}\right)^{b-1+\omega}} \frac{\Gamma(\bar{\zeta}+2.5-\omega)}{\Gamma(\bar{\zeta}+b+1.5)}\left(\frac{m_{r}}{2 k T_{r e f}}\right)^{1 / 2} \frac{\left(E_{c}-E_{a}\right)^{b+\bar{\zeta}+0.5}}{E_{c}^{\bar{\zeta}+1.5-\omega}},
$$

where $a, b$, and $E_{a}$ are the constant values from the Arrhenius form of $a T^{b} \exp \left(E_{a} / k T\right)$. In the present work, $\mathrm{K}_{f}$ of Eq. (25) is adopted for these constants. $\bar{\zeta}$ is the average number of degrees of freedom of the rotational and vibrational energies. In describing the chemical reaction by the TCE and QK models, the VSS and LB models are adopted for the total cross section and rotational and vibrational energy transfer. Then, in the present work, the comparisons of the DSMC calculations are performed for SST+RVT+QCT, $\mathrm{VSS}+\mathrm{LB}+\mathrm{QK}$, and VSS+LB+TCE. In the LB model, the R-V transitions are ignored and the effective collisionnumbers of rotational and vibrational relaxations are determined by Eq. (18).

In Fig. 11, dissociation probabilities of the QCT, QK, and TCE models are compared for various vibrational energy levels of $v=20$ to $v=60$ in the rotational ground state. In this figure, the dissociation 
probability of the QK model is the average over 1,000 particle samplings. In the QCT model, the dissociation probability increases in proportion to the vibrational energy level, and the dissociation probability of $v=60$ is about 0.4 to 0.8 at the relative translational energy of $0.1 \mathrm{eV}$ to $10 \mathrm{eV}$, respectively. However, in the dissociation probabilities of the QK and TCE models, unphysical behavior is observed. The dissociation probability is not in proportion to increasing vibrational energy levels. For example, the dissociation probabilities for $v=50$ to $v=60$ are almost identical.

In Fig. 12, species number density relaxations in equilibrium heat bath calculations for $T=20,000 \mathrm{~K}$ and $T=30,000 K$ by the QCT, QK, and TCE models are presented. In these equilibrium heat bath calculations, the rotational and vibrational energies are frozen for the equilibrium distributions. In the comparisons between the state-resolved QCT model and the previous QK and TCE models, the chemical reactions by the QCT model occur more rapidly than the QK and TCE models. In comparisons between the QK and TCE models, the chemical reactions by QK are slightly faster than the TCE model. The differences of the dissociation probabilities in Fig. 11 account for these results of chemical reactions. At the temperatures of $20,000 \mathrm{~K}$ and $30,000 \mathrm{~K}$, the number densities of high-rotational and vibrational energies are populated, and the dissociation probabilities of the QCT model at these high energy levels are discernably larger than those of the QK and TCE models.

In Fig. 13, relaxations of the rotational and vibration temperatures and species number densities are compared between the DSMC calculations by SST+RVT+QCT, VSS+LB+QK, and VSS+LB+TCE for the C2 and $\mathrm{C} 3$ cases of nonequilibrium heat bath conditions. In figure (a), the rotational and vibration temperatures of $\mathrm{VSS}+\mathrm{LB}+\mathrm{QK}$ and $\mathrm{VSS}+\mathrm{LB}+\mathrm{TCE}$ are discernably over-predicted than those by the SST+RVT+QCT for both $\mathrm{C} 2$ and $\mathrm{C} 3$ cases. However, in figure (b), the relaxation patterns of the species number densities are almost same for the DSMC calculations by the SST+RVT+QCT, VSS+LB+QK, and VSS+LB+QCT. In the equilibrium heat bath calculations, the number density relaxations between these models are discernably different.

In Fig. 14, comparisons of normalized vibrational number density distributions from DSMC calculations by $\mathrm{SST}+\mathrm{RVT}+\mathrm{QCT}$, VSS+LB+QK, and VSS+LB+TCE are presented at $t=1.0 \times 10^{-7} \mathrm{sec}$ and $t=$ $5.1 \times 10^{-8}$ sec for the $\mathrm{C} 2$ and $\mathrm{C} 3$ cases, respectively. In VSS+LB+QK and VSS+LB+TCE, the number densities at high-vibrational energy levels above $v=20$ are significantly more populated than those of $\mathrm{SST}+\mathrm{RVT}+\mathrm{QCT}$ for both cases. The number densities of VSS+LB+QK and VSS+LB+TCE are 11 to 13 times larger than those of SST+RVT+QCT at $v=50$ in the $\mathrm{C} 2$ and $\mathrm{C} 3$ cases. These differences of number densities at high-energy levels make up for the differences of the dissociation probabilities between the QCT and the QK and TCE models, and it produces similar relaxation patterns of species number densities in the nonequilibrium chemical reactions as shown in Fig. 13

2-D cylindrical flows are again calculated by SST+RVT+QCT, VSS+LB+QK, and VSS+LB+TCE. In Fig. 15, comparisons are presented for the temperatures and species number densities along the stagnation line. In figure (a), the rotational temperature is not much higher than the vibrational temperature. The rotational temperature is slightly larger than or similar to the vibrational temperatures in all $\mathrm{SST}+\mathrm{RVT}+\mathrm{QCT}, \mathrm{VSS}+\mathrm{LB}+\mathrm{QK}$, and VSS+LB+TCE simulations. In elastic collisions, the translational temperature of SST+RVT+QCT is higher than that of VSS+LB+QK and VSS+LB+TCE in front of the cylinder. The maximum differences of the translational temperature between the models is about $5,000 \mathrm{~K}$ at the position of $x=17.5 \mathrm{~cm}$. However, the translational temperature of SST+RVT+QCT is smaller than those of VSS+LB+QK and VSS+LB+TCE at distances far from the cylinder, and the temperature gradients of SST+RVT+QCT are steeper than those of VSS+LB+QK and VSS+LB+TCE. The translational, rotational, and vibrational temperatures of VSS+LB+QK are almost the same as those of VSS+LB+TCE. In figure (b), species number densities are compared. In front of the cylinder nose, the number density of dissociated N from SST+RVT+QCT is larger than that of VSS+LB+QK and VSS+LB+TCE, However, it is difficult to clearly see the differences of the chemical reaction models. This is because, in nonequilibrium chemical reactions, the number densities of high-energy levels of VSS+LB+QK and VSS+LB+TCE are significantly more populated than those of SST + RVT + QCT, and this affects the chemical reaction rates.

\section{Summary and Conclusions}

In describing elastic collisions in a state-resolved method, an improved state-specific total cross section (SST) model is proposed in the present work. The SST model is based on the real molecular nature and it describes the total cross section for each rotational and vibrational energy level. The collision parameters of 
the SST model for $\mathrm{N}+\mathrm{N}_{2}$ are calculated by a quasi-classical trajectory method and tabulated in the present work. This SST model is validated by comparisons with the results of master equation calculations.

The state-resolved thermochemistry model is constructed using the SST model and state-to-state transition cross sections from the NASA database for bound-bound and bound-free transitions to analyze the rotation-to-translation (R-T), vibration-to-translation (V-T), and rotation-to-vibration (R-V) energy transfer and the chemical reactions. This first principles approach avoids use of any phenomenological assumptions. In nonequilibrium heat bath calculations, the results of DSMC calculations by the state-resolved model are compared with those of the master equation calculations. The rotational and vibrational nonequilibrium including the R-T, V-T, and R-V transitions are exactly described by the present state-resolved model. In chemical reactions, the state-resolved model accurately describes the quasi-steady state of rotational and vibrational energies, and this model is validated in comparisons with shock-tube experimental data and master equation calculations.

In comparisons between the state-resolved model and the VSS and phenomenological LB model of R-T and V-T transitions, it is observed that the rotational and vibrational temperatures and average energies are over-predicted in comparison to the results from the state-resolved model, and the number densities at high-rotational and vibrational energy levels are significantly more populated than those of the state-resolved model. These number density distributions affect the nonequilibrium chemical reaction rates.

In comparisons between the state-resolved model and the QK and TCE models for chemistry, it is observed that the dissociation probabilities of the state-resolved model are proportional to the vibrational energy levels. However, in the QK and TCE models, unphysical behavior is observed. The increasing dissociation probabilities are not proportional to the increasing of vibrational energy levels. Especially, the dissociation probabilities of $v=50$ to $v=60$ are almost identical. In equilibrium heat bath calculations, it is shown that the chemical reactions by the state-resolved model occur more rapidly than the QK and TCE models, and the chemical reactions by the QK model are slightly faster than those by the TCE model. In nonequilibrium heat bath calculations, the chemical reactions by the state-resolved model and QK model appear similar. This is because, in the phenomenological LB model, the number densities of high-energy levels are discernably larger than those by the state-resolved model, and this makes up the differences of the dissociation probabilities.

In 2-D cylindrical flow calculations, discernable differences of translational temperature and flow velocity are observed between the results of the SST and VSS models. The temperature and velocity gradient by the SST model are much steeper than those by VSS model. In all models and cases, the rotational temperature is not much higher than the vibrational temperature. Especially, in the state-resolved model, the rotational temperature is similar to the vibrational temperature.

\section{Acknowledgments}

The authors gratefully acknowledge funding for this work through Air Force Office of Scientific Research Grant FA-9550-11-1-0309.

\section{References}

\footnotetext{
${ }^{1}$ Sharma, S. P. and Gillespie, W., "Nonequilibrium and Equilibrium Shock Front Radiation Measurement," Journal of Thermophysics and Heat Transfer, Vol. 5, No. 3, 1991, pp. 257-265.

${ }^{2}$ Fujita, K., Sato, S., Abe, T., and Ebinuma, Y., "Experimental Investigation of Air Radiation Behind a Strong Shock Wave," Journal of Thermophysics and Heat Transfer, Vol. 16, No. 1, 2002, pp. 77-82.

${ }^{3}$ Parker, J. G., "Rotational and Vibrational Relaxation in Diatomic Gases," Physics of Fluids, Vol. 2, No. 4, 1959, pp. 449-462.

${ }^{4}$ Lordi, J. A. and Mates, R. E., "Rotational Relaxation in Nonpolar Diatomic Gas," Physics of Fluids, Vol. 13, No. 2, 1970, pp. 291-308.

${ }^{5}$ Bird, G. A., Molecular Gas Dynamics and Direct Simulation of Gas Flows, Oxford Univ. Press, Oxford, 1994.

${ }^{6}$ Jaffe, R., Schwenke, D., Chaban, G., and Huo, W. M., "Vibrational and Rotational Excitation and Relaxation of Nitrogen from Accurate Theoretical Calculation," AIAA paper 2008-1208, 46th AIAA Aerospace Sciences Meeting and Exhibit, Reno, Nevada, 2008.

${ }^{7}$ Jaffe, R., Schwenke, D., and Chaban, G., "Theoretical Analysis of $\mathrm{N}_{2}$ Collisional Dissociation and Rotation-Vibration Energy Transfer," AIAA paper 2009-1569, 47th AIAA Aerospace Sciences Meeting and Exhibit, Orlando, Florida, 2009.

${ }^{8}$ Koura, K. and Matsumoto, H., Variable Soft Sphere Molecular Model for Invers-power-law or Lannard-Jones Potential, Physics of Fluids A, Vol. 3, No. 10, 1991, pp. 2459-2465.
} 
${ }^{9}$ Koura, K. and Matsumoto, H. Variable Soft Sphere Molecular Model for Air Species, Physics of Fluids A, Vol. 4, No. 5, 1992, pp. 1083-1085.

${ }^{10}$ Hassan, H. A. and Hash, D. B., A General Hard Sphere Model for Monte Carlo Simulation, Physics of Fluids A, Vol. 7 , No. 3, 1993, pp. 738-744

${ }^{11}$ Fan, J., A General Soft-sphere Model for Monte Carlo Simulation, Physics of Fluids, Vol. 14, No, 12, 2002, pp. $4399-4405$.

${ }^{12}$ Kim, J. G., Kwon, O. J., Park, C., Modification and Expansion of the General Soft-sphere Model To High Temperature Based on Collision Integrals, Physics of Fluids, Vol. 20, No. 1, 2008, pp. 017105.

${ }^{13}$ Bernstein, R. B., Atom-Molecule Collision Theory: A Guide for the Experimentalist, Plenum, New York, 1979.

${ }^{14}$ Miller, W. H., Dynamics of Molecular Collisions, Plenum, New York, 1976.

${ }^{15}$ Svehla, R. A., "Estimated Viscosities and Thermal Conductivities of Gases at High Temperatures," NASA TR R-132, 1962.

${ }^{16}$ Stallcop, J. R., Partridge, H., and Levin, E., "Effective Potential Energies and Transport Cross Sections for Interactions of Hydrogen and Nitrogen," Physical Review A, Vol. 62, No. 6, 2000, p. 062709.

${ }^{17}$ Park, C. "Review of Chemical-Kinetic Problems of Future NASA Missions, I: Earth Entries," Journal of Thermophysics and Heat Transfer, Vol. 7, No. 3, 1993, pp. 385-398.

${ }^{18}$ Landau, L. and Teller, E., "Theory of Sound Dispersion," Physics Sowjetunion, Vol. 10, 1936, pp. 34-43.

${ }^{19}$ Millikan, R. C. and White, D. R., "Systematics of Vibrational Relaxation," Journal of Chemical Physics, Vol. 39, No. 12, 1963, pp. 3209-3213.

${ }^{20}$ Cary, B., "Shock-Tube Study of the Thermal Dissociation of Nitrogen," Physics of Fluids, Vol. 8, No. 1. 1965, pp. 26-35.

${ }^{21}$ Byron, S., "Shock-Tube Measurement of the Rate of Dissociation of Nitrogen," Journal of Chemical Physics, Vol. 44, No. 4, 1966, pp. 1378-1388.

${ }^{22}$ Appleton, J. P., Steinberg, M., and Liquornik, D. J., "Shock-Tube Study of Nitrogen Dissociation using VacuumUltraviolet Light Absorption," Journal of Chemical Physics, Vol. 48, No. 2, 1968, pp. 599-608.

${ }^{23}$ Hanson, R. K. and Baganoff, D., "Shock-Tube Study of Nitrogen Dissociation Rates Using Pressure Measurements," AIAA Journal, Vol. 10, No. 2, 1972, pp. 211-215.

${ }^{24}$ Bird, G. A., "The Q-K model for gas-phase chemical reaction rates," Physics of Fluids, Vol. 23, No. 10, 2011, pp. 106101. 


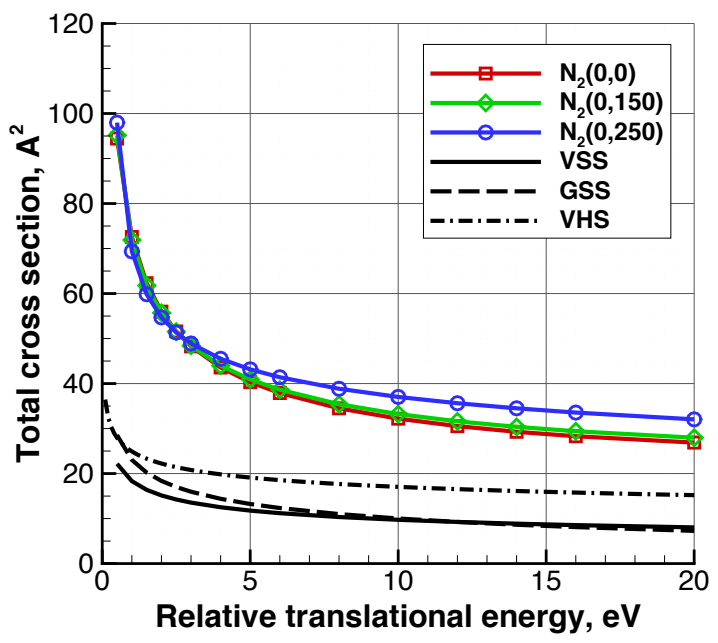

(a) Total cross section for $\mathrm{N}+\mathrm{N}_{2}(0, J)$.

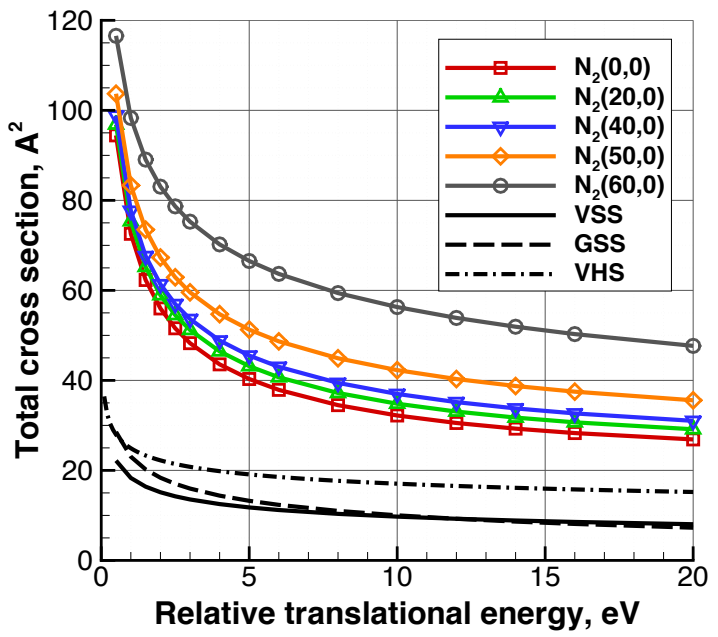

(c) Total cross section for $\mathrm{N}+\mathrm{N}_{2}(v, 0)$.

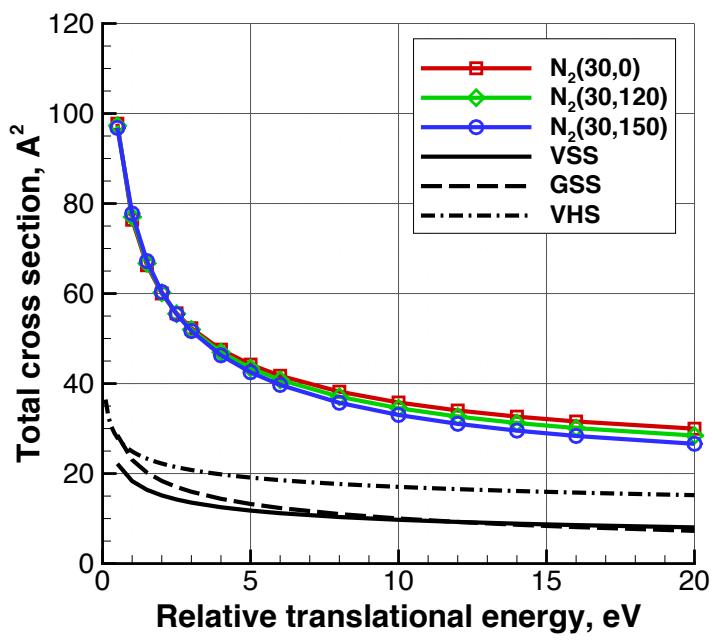

(b) Total cross section for $\mathrm{N}+\mathrm{N}_{2}(30, J)$.

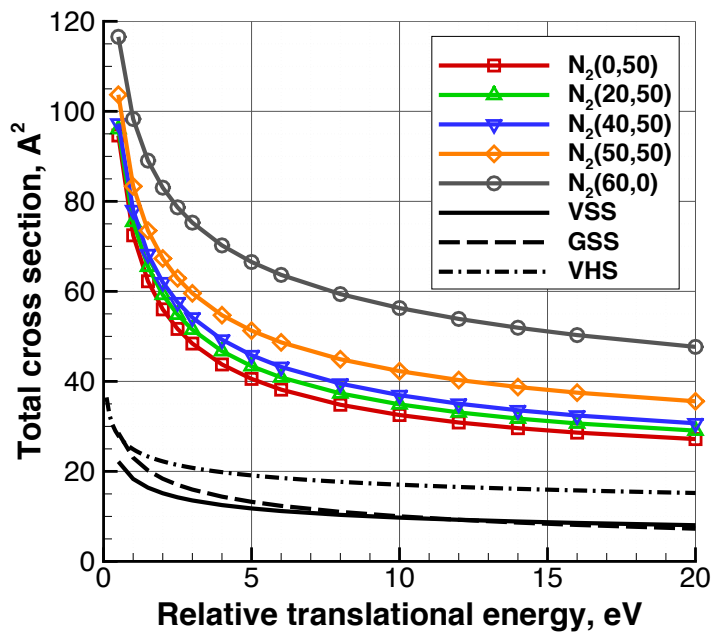

(d) Total cross section for $\mathrm{N}+\mathrm{N}_{2}(v, 50)$.

Figure 1. Comparisons of the total cross sections between the SST model and the previous VHS, VSS, and GSS models. 


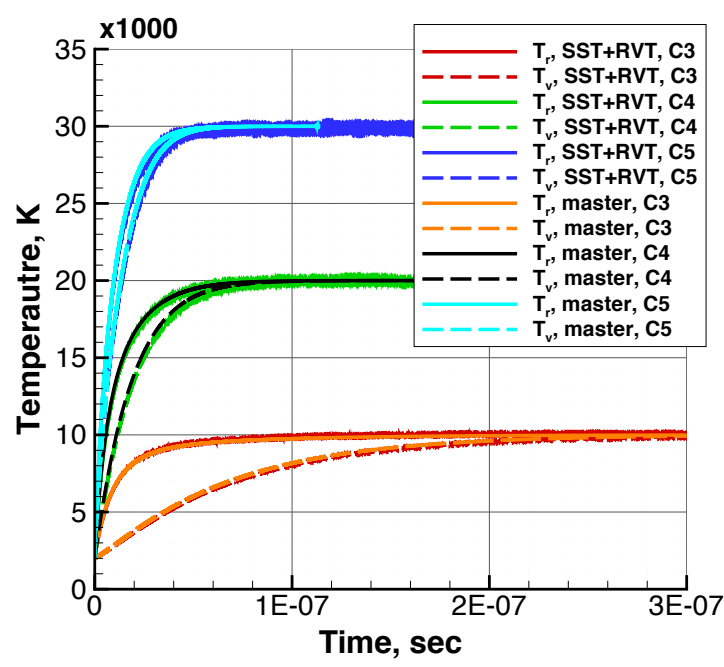

(a) Temperatures.

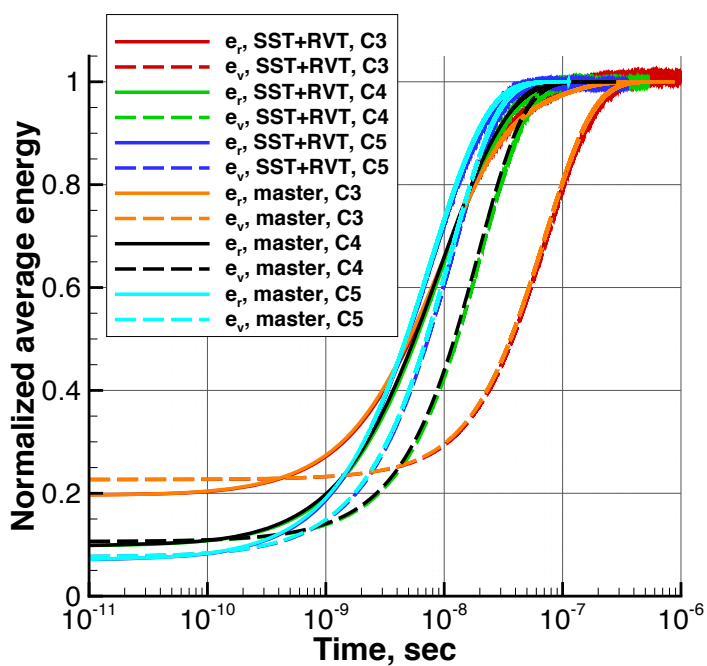

(b) Normalized average energies.

Figure 2. Comparisons of the rotational and vibrational temperatures and normalized average energies between the DSMC calculations by the SST+RVT and master equation calculations.

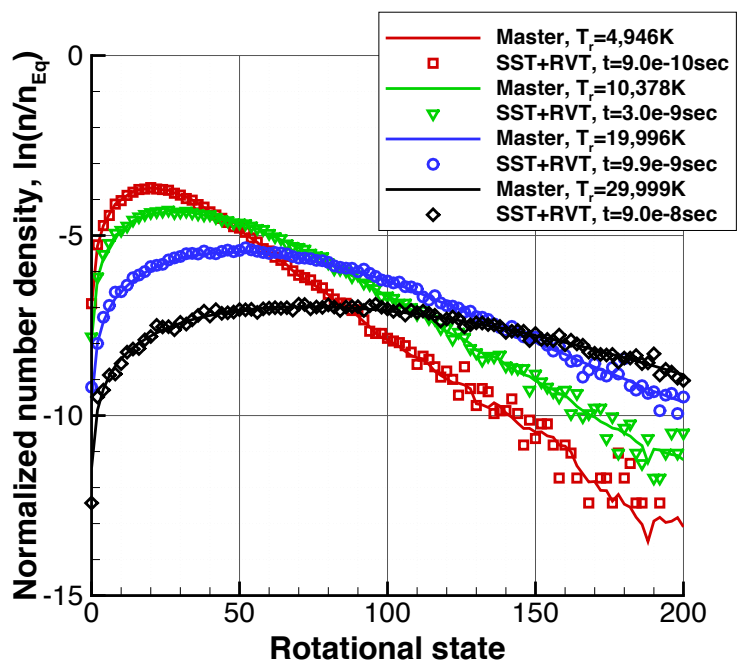

(a) Number density distributions for $\mathrm{N}_{2}(0, J)$.

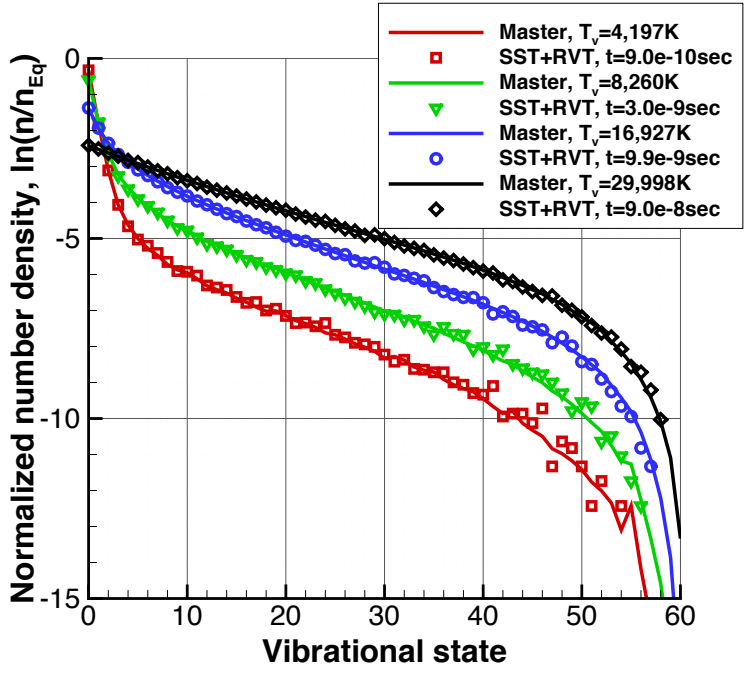

(b) Number density distributions for $\mathrm{N}_{2}(v, 0)$.

Figure 3. Comparisons of the normalized number density distributions for the C5 case between the DSMC calculations by the SST+RVT model and master equation calculations. 


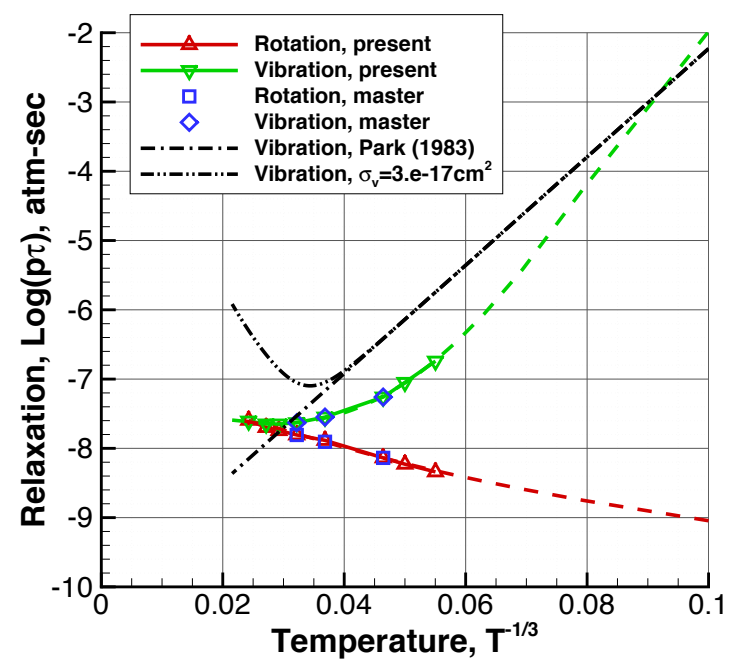

Figure 4. Rotational and vibrational relaxation parameters of $\mathbf{N}+\mathrm{N}_{2}$.

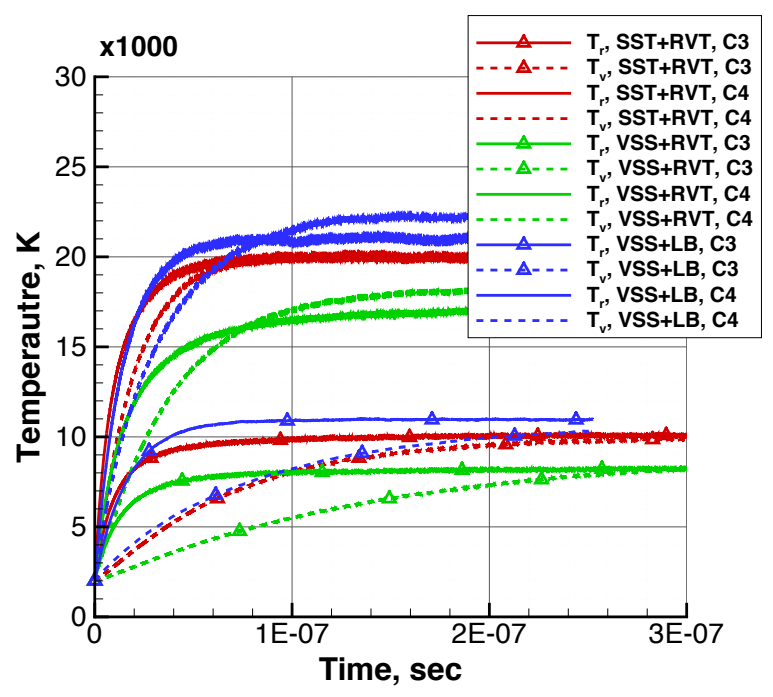

(a) Temperatures.

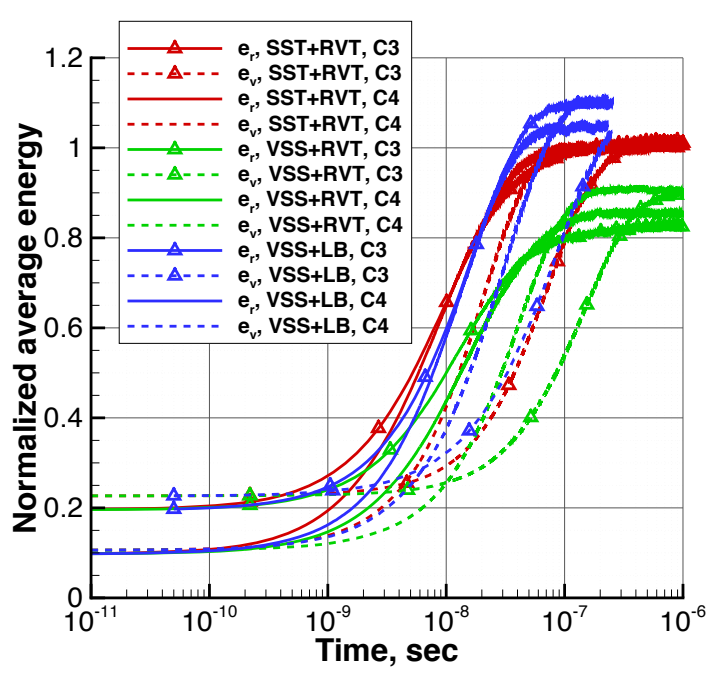

(b) Normalized average energies.

Figure 5. Comparisons of the rotational and vibrational temperatures and normalized average energies between the DSMC calculations by the SST+RVT, VSS+RVT, and VSS+LB models for the C3 and C4 cases. 


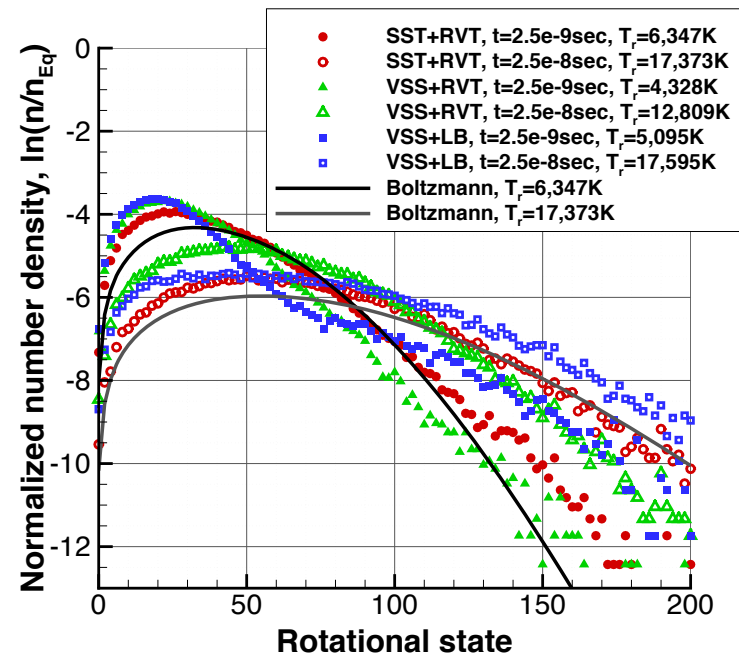

(a) Number density distributions for $\mathrm{N}_{2}(0, J)$.

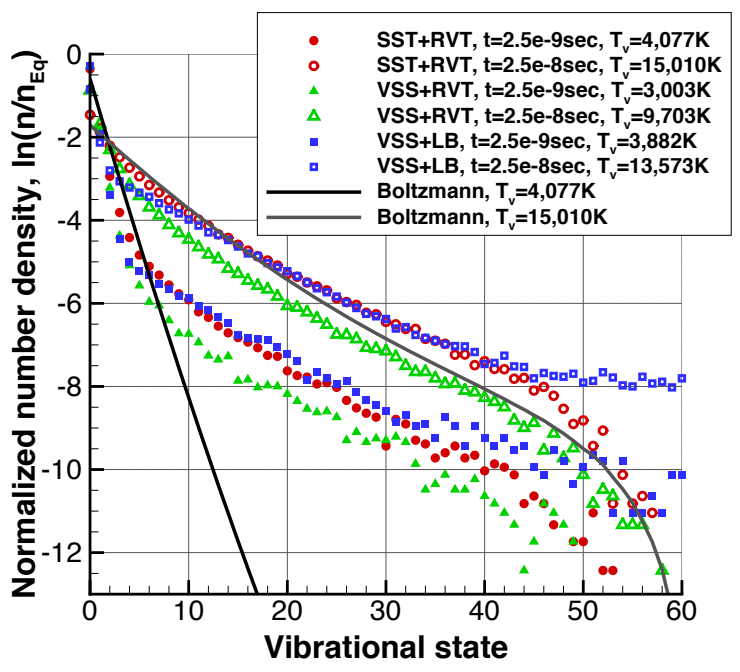

(b) Number density distributions for $\mathrm{N}_{2}(v, 0)$.

Figure 6. Comparisons of the normalized number density distributions for the C4 case between the DSMC calculations using the SST+RVT, VSS+RVT, and VSS+LB models.

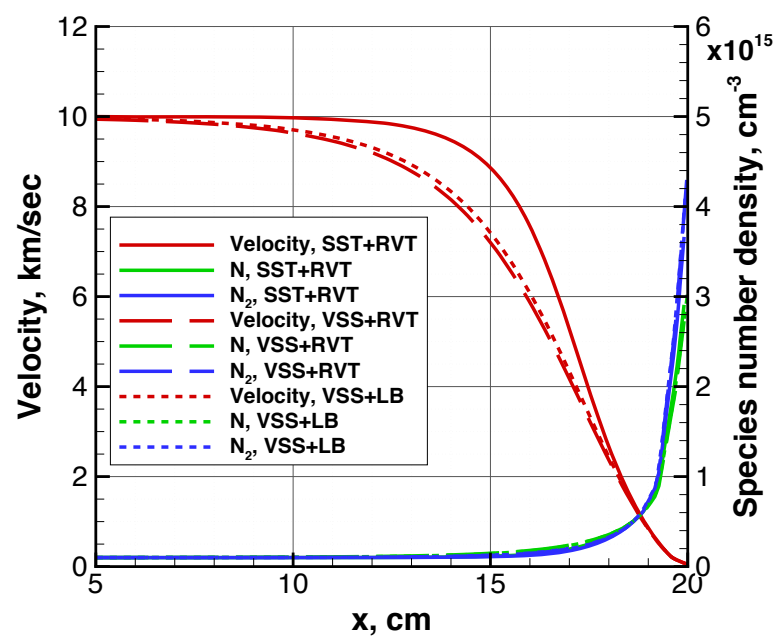

(a) Flow velocity and species number densities.

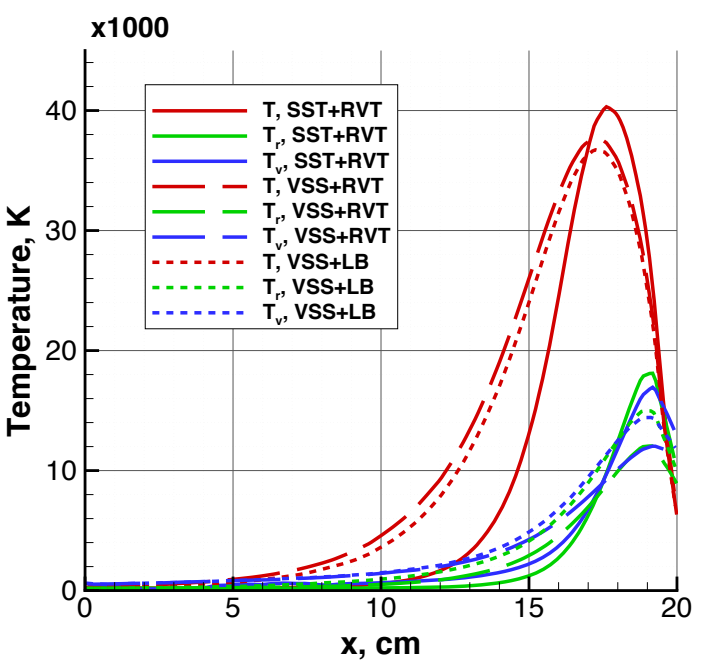

(b) Translational, rotational, and vibrational temperatures.

Figure 7. Comparisons of the flow velocity, species number densities, and temperatures along the stagnation line of a 2-D cylinder. 


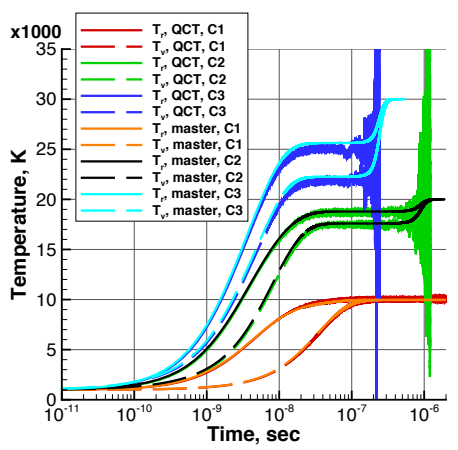

(a) Temperatures.

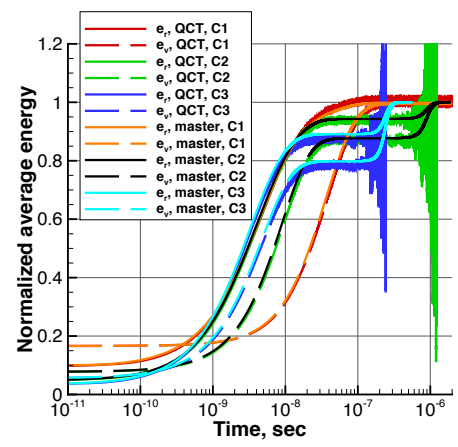

(b) Normalized average energies.

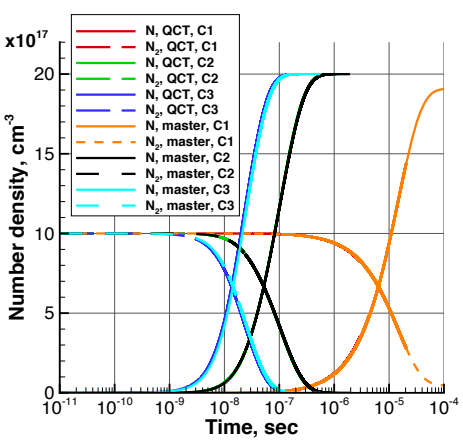

(c) Species number densities.

Figure 8. Comparisons of the rotational and vibrational temperatures, normalized average energies, and species number densities between the DSMC calculations by the SST+RVT+QCT model and master equation calculations.

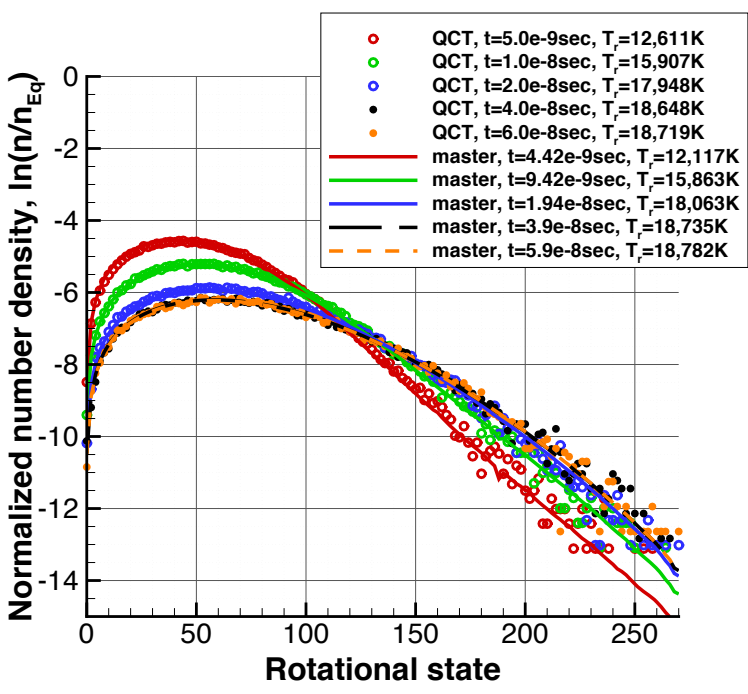

(a) Number density distributions for $\mathrm{N}_{2}(0, J)$.

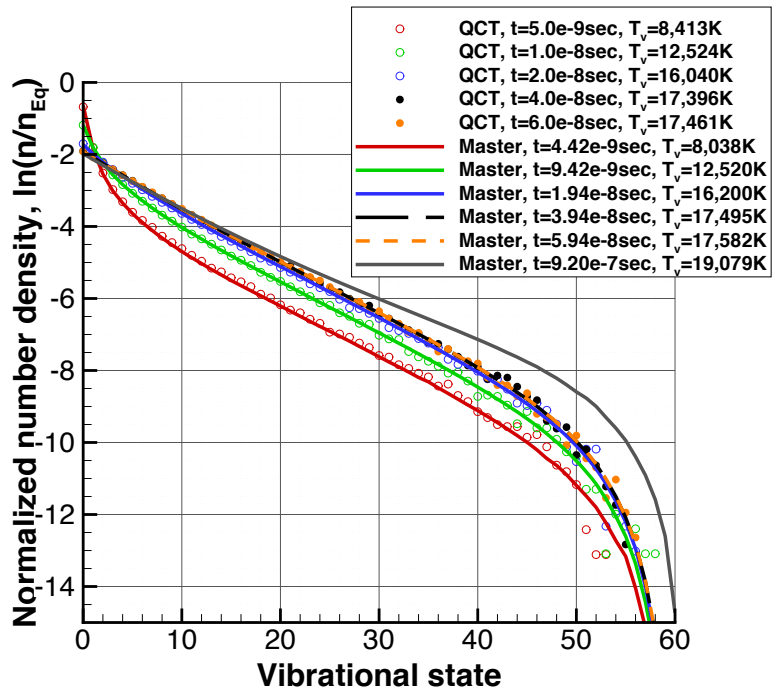

(b) Number density distributions for $\mathrm{N}_{2}(v, 0)$.

Figure 9. Comparisons of the normalized number density distributions of the C2 case between the DSMC calculation by the SST+RVT+QCT model and master equation calculations. 


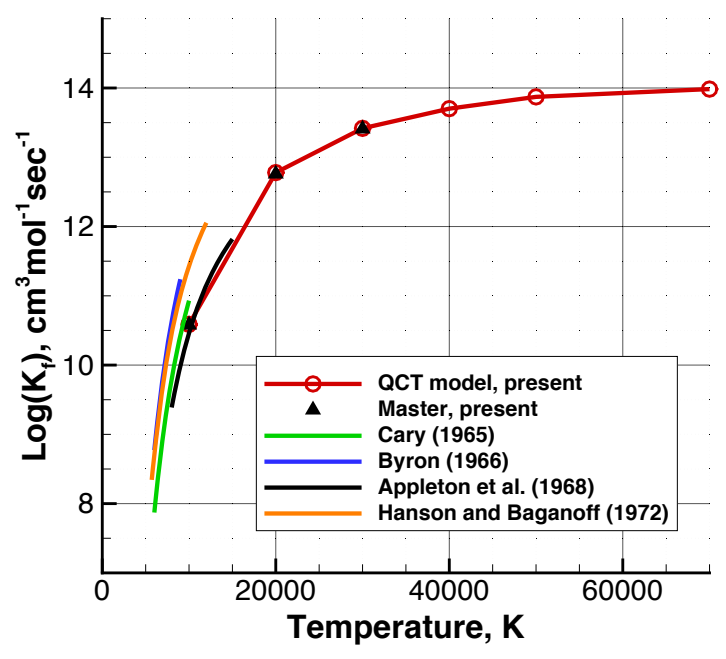

Figure 10. Comparisons of the calculated dissociation reaction rates by SST+RVT+QCT with the shock-tube measured data. ${ }^{20-23}$

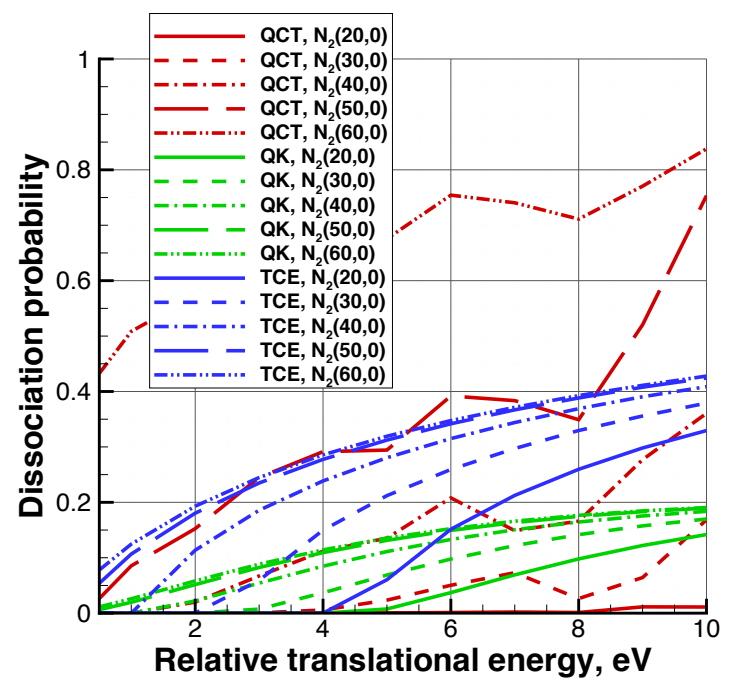

Figure 11. Dissociation probabilities of the QCT, QK, and TCE models for various vibrational levels. 


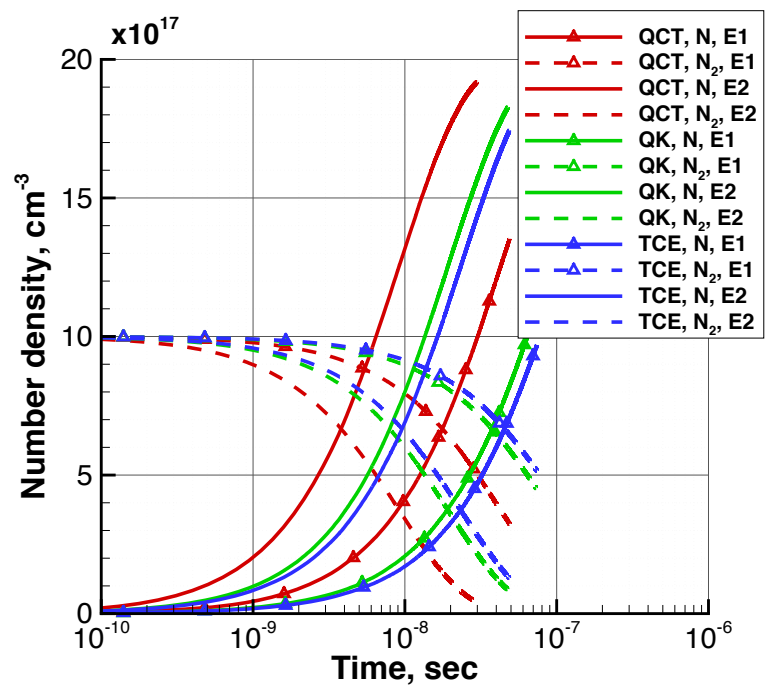

Figure 12. Species number density relaxations in equilibrium heat conditions of $T=20,000 K(\mathrm{E} 1)$ and $30,000 K(\mathbf{E} 2)$.

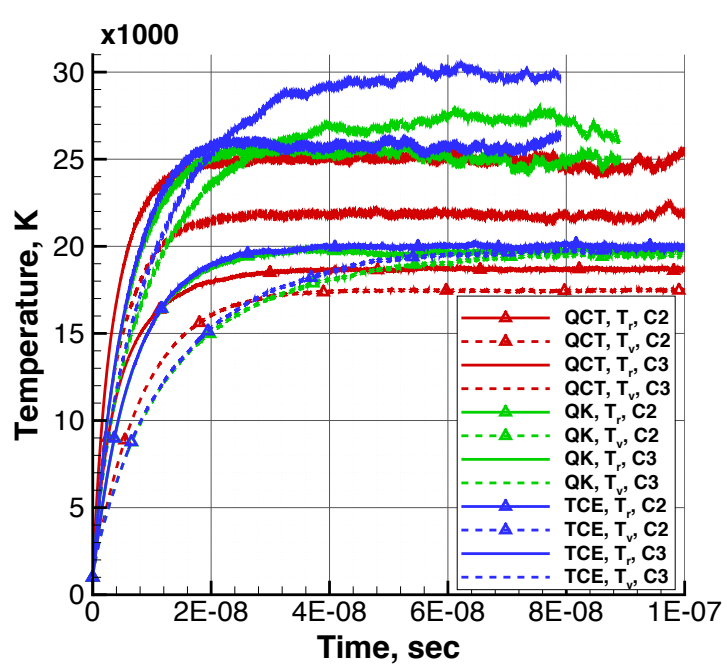

(a) Temperatures.

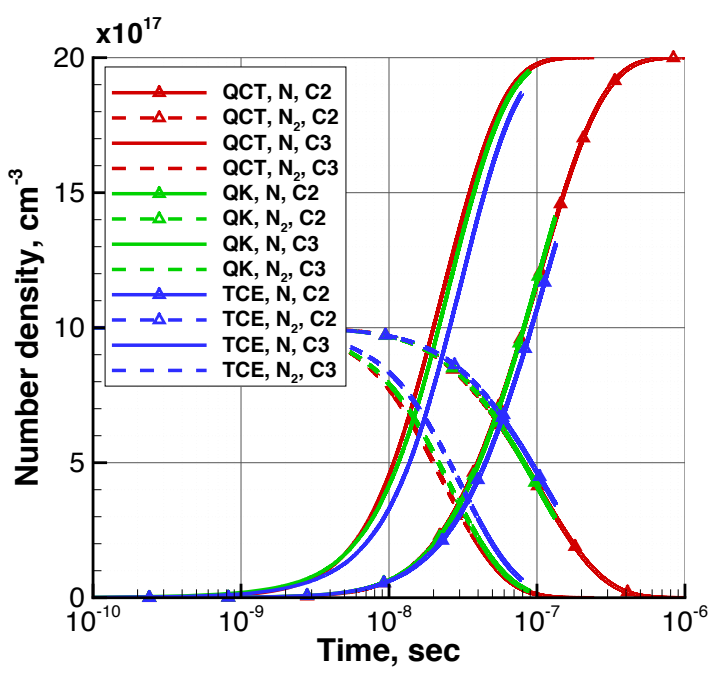

(b) Species number densities.

Figure 13. Comparisons of the rotational and vibrational temperatures and species number densities of the C2 and C3 cases between the DSMC calculations by the SST+RVT+QCT, VSS+LB+QK, and VSS+LB+TCE models. 


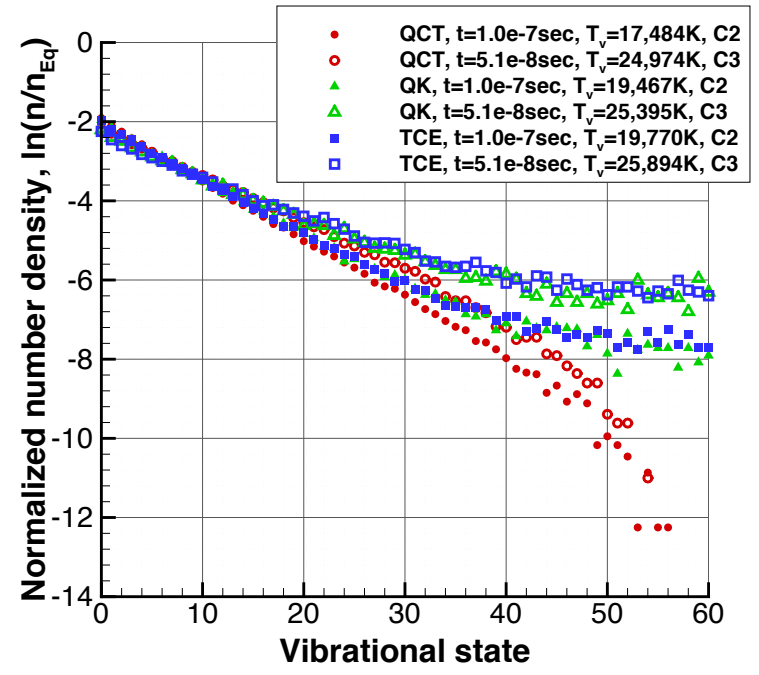

Figure 14. Comparisons of the normalized number density distribution of $\mathbf{N}_{2}(v, 0)$ in the C2 and C3 cases between the DSMC calculations of the SST+RVT+QCT, VSS+LB+QK, and VSS+LB+TCE models.

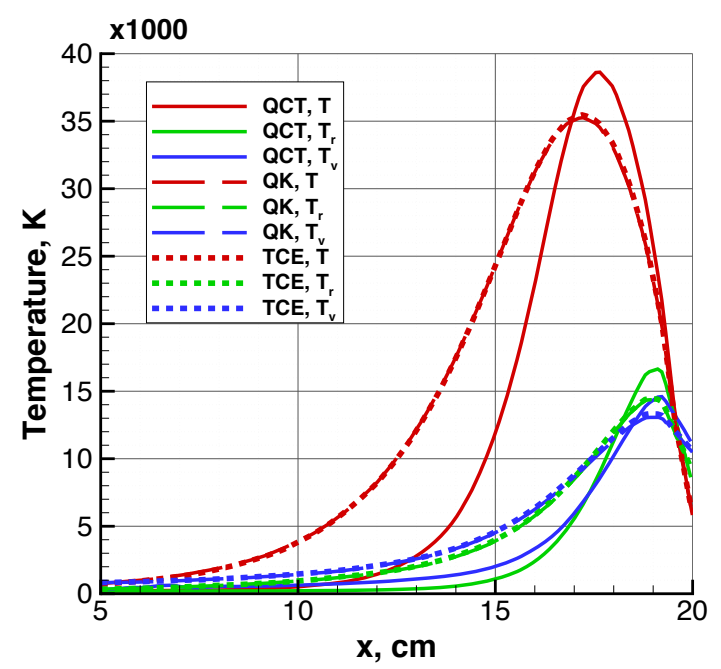

(a) Translational, rotational, and vibrational temperatures.

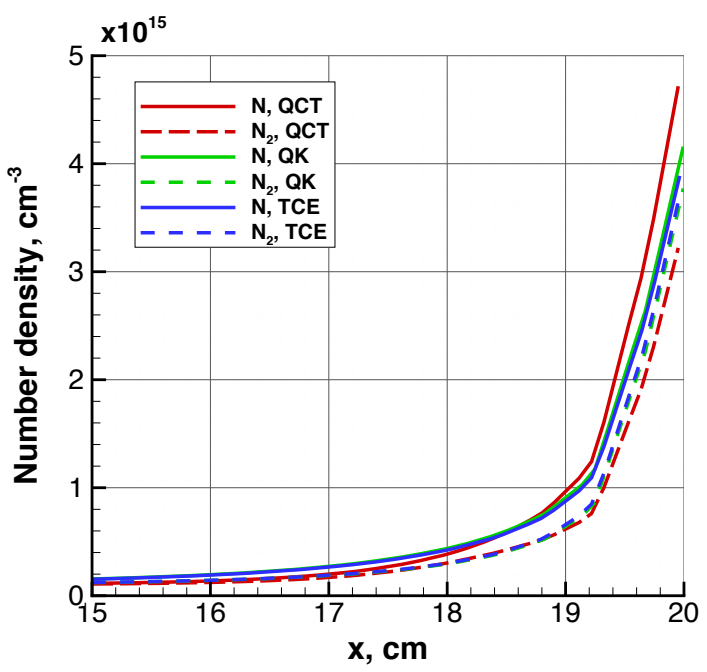

(b) Species number densities.

Figure 15. Comparisons of the temperatures and species number densities between the DSMC calculations of the SST+RVT+QCT, VSS+LB+QK, and VSS+LB+TCE models along the stagnation line of the 2-D cylinder. 


\section{Appendix}

Table 4. Collision parameters of the SST model.

\begin{tabular}{|c|c|c|c|c|c|}
\hline & $a_{1}$ & $a_{2}$ & $a_{3}$ & $a_{4}$ & $a_{5}$ \\
\hline 0 & $-3.3030 \mathrm{E}+01$ & $1.7163 \mathrm{E}-03$ & $1.8815 \mathrm{E}-01$ & $8.1313 \mathrm{E}-01$ & $-2.6237 \mathrm{E}-01$ \\
\hline 1 & & & & & \\
\hline 2 & -3 & & & & \\
\hline 3 & & & & & \\
\hline 4 & -3 & & & & \\
\hline 5 & & 3 & & 00 & \\
\hline 6 & 01 & -2.3567 & 8.540 & +00 & \\
\hline 7 & $\mathrm{E}+01$ & -3.028 & 7.3 & 00 & 01 \\
\hline 8 & & -3.890 & 4.9 & & \\
\hline 9 & $\mathrm{E}+01$ & -4.1529 & 4.228 & 00 & 01 \\
\hline 10 & & -4.74 & & & \\
\hline 11 & -3. & -4.7 & & & \\
\hline 12 & & & & & \\
\hline 13 & & & & & \\
\hline 14 & & & & & \\
\hline 15 & & & & & \\
\hline 16 & +01 & -5. & 3.1 & & \\
\hline 17 & & & & & \\
\hline 18 & $-3.3338 \mathrm{E}+01$ & $-5.8612 \mathrm{E}-03$ & $5.7788 \mathrm{E}-03$ & 1.2370 & -3.630 \\
\hline 19 & $-3.3342 \mathrm{E}+01$ & -6.4232 & & & \\
\hline 20 & $-3.3362 \mathrm{E}+01$ & $-6.7667 \mathrm{E}-03$ & $-8.0550 \mathrm{E}-03$ & $1.2720 \mathrm{E}+00$ & -3.719 \\
\hline 21 & & & & & \\
\hline 22 & $-3.3370 \mathrm{E}+01$ & $-7.0294 \mathrm{E}-03$ & -1.211 & $1.2893 \mathrm{E}+00$ & -3.77 \\
\hline 23 & & & & & \\
\hline 24 & $-3.3351 \mathrm{E}+01$ & $-7.2785 \mathrm{E}-03$ & -6.616 & $1.2639 \mathrm{E}+00$ & -3.709 \\
\hline 25 & & & & & \\
\hline 26 & & $-7.4354 \mathrm{E}-03$ & & $1.2428 \mathrm{E}+00$ & \\
\hline 27 & $-3.3295 \mathrm{E}+01$ & $-6.8336 \mathrm{E}-03$ & $1.6909 \mathrm{E}-02$ & $1.1773 \mathrm{E}+00$ & $-3.4422 \mathrm{E}-01$ \\
\hline 28 & $-3.3267 \mathrm{E}+01$ & $-6.2752 \mathrm{E}-03$ & $3.1438 \mathrm{E}-02$ & $1.1360 \mathrm{E}+00$ & $-3.3304 \mathrm{E}-01$ \\
\hline 29 & $-3.3269 \mathrm{E}+01$ & $-7.0434 \mathrm{E}-03$ & 2.7149E-02 & $1.1384 \mathrm{E}+00$ & $-3.3233 \mathrm{E}-01$ \\
\hline 30 & $-3.3244 \mathrm{E}+01$ & & & & $-3.2611 \mathrm{E}-01$ \\
\hline
\end{tabular}


Table 5. Collision parameters of the SST model. (continued)

\begin{tabular}{|c|c|c|c|c|c|}
\hline & $a_{1}$ & $a_{2}$ & $a_{3}$ & $a_{4}$ & $a_{5}$ \\
\hline 31 & $-3.3199 \mathrm{E}+01$ & $-5.7521 \mathrm{E}-03$ & $6.4015 \mathrm{E}-02$ & $1.0427 \mathrm{E}+00$ & $-3.0762 \mathrm{E}-01$ \\
\hline 32 & $-3.3130 \mathrm{E}+01$ & $-4.6922 \mathrm{E}-03$ & $9.7043 \mathrm{E}-02$ & $9.4909 \mathrm{E}-01$ & $-2.8386 \mathrm{E}-01$ \\
\hline 33 & $-3.3105 \mathrm{E}+01$ & $-4.3020 \mathrm{E}-03$ & $1.1020 \mathrm{E}-01$ & $9.1376 \mathrm{E}-01$ & $-2.7415 \mathrm{E}-01$ \\
\hline 34 & $-3.3053 \mathrm{E}+01$ & $-3.1836 \mathrm{E}-03$ & $1.3812 \mathrm{E}-01$ & $8.4356 \mathrm{E}-01$ & $-2.5837 \mathrm{E}-01$ \\
\hline 35 & $-3.2963 \mathrm{E}+01$ & $-1.8095 \mathrm{E}-03$ & $1.8145 \mathrm{E}-01$ & 7.0467E-01 & $-2.1776 \mathrm{E}-01$ \\
\hline 36 & $-3.2906 \mathrm{E}+01$ & $-3.8833 \mathrm{E}-04$ & $2.1542 \mathrm{E}-01$ & $6.3578 \mathrm{E}-01$ & $-2.0412 \mathrm{E}-01$ \\
\hline 37 & $-3.2845 \mathrm{E}+01$ & 7.1534E-04 & $2.4689 \mathrm{E}-01$ & $5.5564 \mathrm{E}-01$ & $-1.8379 \mathrm{E}-01$ \\
\hline 38 & $-3.2756 \mathrm{E}+01$ & $3.1149 \mathrm{E}-03$ & $2.9736 \mathrm{E}-01$ & $4.2920 \mathrm{E}-01$ & $-1.5218 \mathrm{E}-01$ \\
\hline 39 & $-3.2735 \mathrm{E}+01$ & 4.1964E-03 & $3.1409 \mathrm{E}-01$ & 4.0853E-01 & $-1.4986 \mathrm{E}-01$ \\
\hline 40 & $-3.2694 \mathrm{E}+01$ & $5.2789 \mathrm{E}-03$ & $3.3740 \mathrm{E}-01$ & $3.5646 \mathrm{E}-01$ & $-1.3803 \mathrm{E}-01$ \\
\hline 41 & $-3.2684 \mathrm{E}+01$ & $5.7107 \mathrm{E}-03$ & $3.4152 \mathrm{E}-01$ & $3.4285 \mathrm{E}-01$ & $-1.3328 \mathrm{E}-01$ \\
\hline 42 & $-3.2648 \mathrm{E}+01$ & 6.0407E-03 & 3.5337E-01 & $3.0118 \mathrm{E}-01$ & $-1.2490 \mathrm{E}-01$ \\
\hline 43 & $-3.2585 \mathrm{E}+01$ & $6.5111 \mathrm{E}-03$ & $3.7104 \mathrm{E}-01$ & $1.9941 \mathrm{E}-01$ & $-9.0316 \mathrm{E}-02$ \\
\hline 44 & $-3.2581 \mathrm{E}+01$ & $6.1109 \mathrm{E}-03$ & $3.6408 \mathrm{E}-01$ & $1.9161 \mathrm{E}-01$ & $-8.5555 \mathrm{E}$ \\
\hline 45 & $-3.2553 \mathrm{E}+01$ & $6.5891 \mathrm{E}-03$ & $3.7087 \mathrm{E}-01$ & $1.6670 \mathrm{E}-01$ & $-8.1435 \mathrm{E}-02$ \\
\hline 46 & $-3.2543 \mathrm{E}+01$ & $6.5155 \mathrm{E}-03$ & $3.6856 \mathrm{E}-01$ & $1.4613 \mathrm{E}-01$ & $-7.4211 \mathrm{E}-02$ \\
\hline 47 & $-3.2548 \mathrm{E}+01$ & $6.0417 \mathrm{E}-03$ & 3.5633E-01 & $1.6187 \mathrm{E}-01$ & $-7.5415 \mathrm{E}-02$ \\
\hline 48 & $-3.2560 \mathrm{E}+01$ & $5.3335 \mathrm{E}-03$ & $3.4187 \mathrm{E}-01$ & $1.7987 \mathrm{E}-01$ & $-7.8565 \mathrm{E}-02$ \\
\hline 49 & $-3.2489 \mathrm{E}+01$ & $6.7078 \mathrm{E}-03$ & $3.6726 \mathrm{E}-01$ & $7.7610 \mathrm{E}-02$ & $-4.7907 \mathrm{E}-02$ \\
\hline 50 & $-3.2515 \mathrm{E}+01$ & $5.4610 \mathrm{E}-03$ & $3.3978 \mathrm{E}-01$ & $1.2509 \mathrm{E}-01$ & $-6.0110 \mathrm{E}-02$ \\
\hline 51 & $-3.2495 \mathrm{E}+01$ & $4.8258 \mathrm{E}-03$ & $3.3337 \mathrm{E}-01$ & $1.0583 \mathrm{E}-01$ & $-5.3673 \mathrm{E}-02$ \\
\hline 52 & $-3.2455 \mathrm{E}+01$ & $5.6209 \mathrm{E}-03$ & $3.4158 \mathrm{E}-01$ & $5.5713 \mathrm{E}-02$ & $-3.8153 \mathrm{E}-02$ \\
\hline 53 & $-3.2459 \mathrm{E}+01$ & $4.7662 \mathrm{E}-03$ & $3.2149 \mathrm{E}-01$ & $5.5237 \mathrm{E}-02$ & $-3.0712 \mathrm{E}-02$ \\
\hline 54 & $-3.2423 \mathrm{E}+01$ & $3.8803 \mathrm{E}-03$ & $3.1543 \mathrm{E}-01$ & $1.6918 \mathrm{E}-02$ & $-1.8758 \mathrm{E}-02$ \\
\hline 55 & $-3.2353 \mathrm{E}+01$ & 4.1713E-03 & $3.2592 \mathrm{E}-01$ & $-7.6810 \mathrm{E}-02$ & $1.0719 \mathrm{E}-02$ \\
\hline 56 & $-3.2313 \mathrm{E}+01$ & 4.1087E-03 & $3.2195 \mathrm{E}-01$ & $-1.3991 \mathrm{E}-01$ & $3.8945 \mathrm{E}-02$ \\
\hline 57 & $-3.2341 \mathrm{E}+01$ & $2.5161 \mathrm{E}-03$ & $2.8382 \mathrm{E}-01$ & $-6.8041 \mathrm{E}-02$ & $2.0518 \mathrm{E}-02$ \\
\hline 58 & $-3.2343 \mathrm{E}+01$ & $1.7842 \mathrm{E}-03$ & $2.6264 \mathrm{E}-01$ & $-8.8439 \mathrm{E}-03$ & $1.3293 \mathrm{E}-03$ \\
\hline 59 & $-3.2290 \mathrm{E}+01$ & 6.3533E-04 & $2.5170 \mathrm{E}-01$ & $-2.7241 \mathrm{E}-02$ & $1.0358 \mathrm{E}-02$ \\
\hline 60 & $-3.2191 \mathrm{E}+01$ & $1.4417 \mathrm{E}-03$ & $2.7254 \mathrm{E}-01$ & $-1.0866 \mathrm{E}-01$ & $3.2535 \mathrm{E}-02$ \\
\hline
\end{tabular}

